OPEN ACCESS

Edited by:

Xi-Nian Zuo,

Institute of Psychology (CAS), China

Reviewed by:

Alle Meije Wink,

VU University Medical Center,

Netherlands

Miao Cao,

Beijing Normal University, China

*Correspondence:

Haiyun L

haiyunli@ccmu.edu.cn

Specialty section: This article was submitted to Brain Imaging Methods, a section of the journal

Frontiers in Neuroscience

Received: 04 September 2017

Accepted: 15 January 2018

Published: 02 February 2018

Citation:

Dong J, Jing B, Ma X, Liu H, Mo X and Li H (2018) Hurst Exponent Analysis of Resting-State fMRI Signal Complexity across the Adult Lifespan.

Front. Neurosci. 12:34 doi: 10.3389/fnins.2018.00034

\section{Hurst Exponent Analysis of Resting-State fMRI Signal Complexity across the Adult Lifespan}

\author{
Jianxin Dong ${ }^{1,2}$, Bin Jing ${ }^{1}$, Xiangyu Ma ${ }^{1}$, Han Liu ${ }^{1}$, Xiao $\mathrm{Mo}^{1}$ and Haiyun $\mathrm{Li}^{1 *}$ \\ ${ }^{1}$ School of Biomedical Engineering, Capital Medical University, Beijing, China, ${ }^{2}$ Yanjing Medical College, Capital Medical \\ University, Beijing, China
}

Exploring functional information among various brain regions across time enables understanding of healthy aging process and holds great promise for age-related brain disease diagnosis. This paper proposed a method to explore fractal complexity of the resting-state functional magnetic resonance imaging ( $r s-\mathrm{fMRI}$ ) signal in the human brain across the adult lifespan using Hurst exponent (HE). We took advantage of the examined rs-fMRI data from 116 adults 19 to 85 years of age (44.3 \pm 19.4 years, 49 females) from $\mathrm{NKI} /$ Rockland sample. Region-wise and voxel-wise analyses were performed to investigate the effects of age, gender, and their interaction on complexity. In region-wise analysis, we found that the healthy aging is accompanied by a loss of complexity in frontal and parietal lobe and increased complexity in insula, limbic, and temporal lobe. Meanwhile, differences in HE between genders were found to be significant in parietal lobe $(p=0.04$, corrected). However, there was no interaction between gender and age. In voxel-wise analysis, the significant complexity decrease with aging was found in frontal and parietal lobe, and complexity increase was found in insula, limbic lobe, occipital lobe, and temporal lobe with aging. Meanwhile, differences in HE between genders were found to be significant in frontal, parietal, and limbic lobe. Furthermore, we found age and sex interaction in right parahippocampal gyrus ( $p=0.04$, corrected). Our findings reveal HE variations of the rs-fMRI signal across the human adult lifespan and show that HE may serve as a new parameter to assess healthy aging process.

Keywords: hurst exponent, complexity, healthy aging, lifespan, resting-state fMRI

\section{INTRODUCTION}

As the elderly population increases, age-related cognitive changes across healthy lifespan emerges as a major concern which can interfere with daily routines and has an impact on quality of life (Hedden and Gabrieli, 2004; St John and Montgomery, 2010; Abrahamson et al., 2012). There is thus a need of more profound comprehension of the law of brain functional changes associated with healthy aging.

Functional magnetic resonance imaging (fMRI) provides non-invasive techniques to explore aging human brain in vivo (Bandettini, 2007; Grady, 2008; Dosenbach et al., 2010; Uddin et al., 2010). At present, fMRI study is generally based on task or resting state. Resting state studies of spontaneous fluctuations in fMRI signals have demonstrated huge potential in mapping the brain's intrinsic functional features (Kruger and Glover, 2001; Yan et al., 2009). Ciuciu et al. found that spontaneous brain activities had scale-free dynamics (Ciuciu et al., 2012). He suggested that 
brain activity observed at rs-fMRI signals exhibits a $1 /$ f-like power spectrum, and the irregular brain activity contributing to this " $1 /$ f slope" of the power spectrum was scale-free brain activity (He, 2014). And "scale-free" is the equivalent terminologies for "self-similar" (Expert et al., 2011). Thus, Hurst exponent (HE) has attracted researchers' attention on assessment of spontaneous signal fluctuations in fMRI due to its well displaying the scalefree dynamics by representing the self-similarity of a time series (Maxim et al., 2005; Park et al., 2010). Wink et al. utilized HE to quantify fractal complexity and describe pathological and physiological features, then found that HE increased in bilateral hippocampus with healthy aging (Wink et al., 2006). Liu et al. (2013) suggested that the fractal complexity of resting state BOLD time-series may provide a viable measure to probe the complexity of the underlying brain activity and they found a trend of decreasing complexity of brain endogenous oscillations measured by mean approximate entropy in gray matter with healthy aging. Here complexity can be described as the presence of similar patterns in the rs-fMRI signal. Lipsitz (2004) found that complexity of physiological signals decreased with aging. Further characterizing resting-state brain activity across time with $\mathrm{HE}$ analysis could provide new insights into healthy aging process.

In addition, brain healthy aging may differ between genders. Some studies found gender-related differences in prefrontal and limbic regions with emotional and cognitive tasks (Boghi et al., 2006; Hofer et al., 2006; Mcrae et al., 2008; Schulterüther et al., 2008; Keller and Menon, 2009). Ni et.al explored age and gender effects using multifractal analysis of the rs-fMRI series in default mode network (Ni et al., 2014). Lopez-Larson et al. (2011) sought to assess the effects of age and gender, by measuring local brain connectivity of healthy controls using rs-fMRI data. They found that there existed decreased regional homogeneity with aging, and the fastest decline was in the temporal lobe and anterior cingulate. However, their sample size was not large enough to support their findings and using Kendall's coefficient of concordance to compute regional homogeneity values may be sensitive to random noises.

In this study, we proposed a HE based method to detect fractal complexity of the rs-fMRI signal in the human brain across the adult lifespan. Adopting a large sample, we further investigated whether there exist gender difference and interaction between gender and age in HE.

\section{MATERIALS AND METHODS}

\section{Subjects}

Images used in the study are from the Nathan Kline Institute/Rockland Sample (NKI-RS) (Nooner et al., 2012), which is publicly available at the International Neuroimaging Datasharing Initiative online database. The initial release of the NKI-RS included 207 participants. After excluding subjects with diagnosed mental disorders, who all underwent multimodal brain scans and a battery of psychiatric assessments, 116 healthy subjects with mean age of 44.3 years (age range: 19-85 years, $S D=19.4$, median $=43,67$ males and 49 females) were selected. Demographic characteristics of the subjects are displayed in Table 1.
TABLE 1 | Demographic characteristics of the participants.

\begin{tabular}{lccc}
\hline Characteristics & Males & Females & Significance (p-values) \\
\hline subjects & $67(57.8 \%)$ & $49(42.2 \%)$ & - \\
Age, years & $42.5 \pm 18.0$ & $46.8 \pm 21.2$ & 0.25 \\
\hline
\end{tabular}

Participants all went through a scan session utilizing a Siemens Tim Trio 3.0 T 8 channel MRI scanner. All participants were instructed to keep their eyes closed, relax their minds, and not move during the scanning. Each subject completed a $650 \mathrm{~s}$ rs-fMRI scan and then a scan session comprised 260 functional volumes. Rs-fMRI scans were collected using an echo-planar imaging sequence [time echo $(\mathrm{TE})=30 \mathrm{~ms}$, time repetition (TR) $=2.5 \mathrm{~s}$, field of view $(\mathrm{FOV})=216 \times 216 \mathrm{~mm}^{2}$, flip angle $(\mathrm{FA})=$ $80^{\circ}$, matrix size $=64 \times 64$, number of slices $=38$, voxel size $=$ $3.0 \times 3.0 \times 3.0 \mathrm{~mm}^{3}, 260$ volumes]. Each image was viewed to ensure that the whole brain was covered.

\section{Data Preprocessing}

All the preprocessing was performed utilizing the Data Processing Assistant for Resting-State fMRI (DPARSF ${ }^{1}$, Yan and Zang, 2010) which is based on Statistical Parametric Mapping $\left(\mathrm{SPM}^{2}\right)$ and Resting-State fMRI Data Analysis Toolkit (REST ${ }^{3}$, Song et al., 2011).

Preprocessing of rs-fMRI images included the following: (i) discarding of the first 10 volumes from each scan for signal equilibration and to make the subjects adapted to the environment, (ii) correcting for temporal shifts in fMRI data acquisition (slice timing correction), (iii) correction for head motion, and participant with head motion $>2$ degree of rotation, or $>2 \mathrm{~mm}$ of translation in any direction was excluded, (iv) the Friston-24 model (Friston et al., 1996) was used to regress out head motion effects based on Yan et al.'s (2013) study, and then the signals from white matter and cerebrospinal fluid were regressed out to reduce respiratory and cardiac effects (Fox and Raichle, 2007). Final step is (v) spatially smooth the result data using a $6 \mathrm{~mm}$ full width at half maximum (FWHM) Gaussian kernel. Since spontaneous activities may persist in higher frequency bands (Chen and Glover, 2015), temporal filtering was excluded.

\section{Calculation of HE}

The HE is a scalar which measures long-range correlations of a time series. Rescaled Range (R/S) analysis, which is the most common (Hurst, 1951), can effectively examine the temporal complexity of a time series. In this study, we use R/S analysis for HE calculation.

The principle of $\mathrm{R} / \mathrm{S}$ analysis is demonstrated as follows: given a time series $T$ whose length is $L$, and then $T$ is divided into $N$ intervals and the length of each interval is $A(1 \leq A \leq N)$, $A \times N=L$. The $i$-th interval is marked with $I_{i}$ and the $j$-th element

\footnotetext{
${ }^{1}$ http://www.restfmri.net

${ }^{2}$ http://www.fil.ion.ucl.ac.uk/spm

${ }^{3}$ http://www.restfmri.net
} 
in $I_{i}$ is marked with $x_{i, j}, j=1,2,3 \ldots A$, and $e_{i}$ is the mean value in $I_{i}$ interval, so

$$
\begin{aligned}
y_{i, j} & =\sum_{a=1}^{j}\left(x_{i, a}-e_{i}\right), \quad j=1,2,3 \ldots A \\
R_{i} & =\max _{1 \leq j \leq A}\left\{y_{i, j}\right\}-\min _{1 \leq j \leq A}\left\{y_{i, j}\right\} \\
S_{i} & =\left[\frac{1}{A} \sum_{a=1}^{A}\left(x_{i, a}-e_{i}\right)^{2}\right]^{\frac{1}{2}} \\
(R / S)_{A} & =\frac{1}{N} \sum_{i=1}^{A}\left(R_{i} / S_{i}\right)=c A^{H E}
\end{aligned}
$$

Where $\mathrm{c}$ is a constant and HE was defined as the slope of the line fitting the pairs $\left(\ln A, \ln (R / s)_{A}\right)$ in a least-square sense. The calculation was implemented by home-made scripts with Matlab 2012 (MathWorks, Natick, MA).

$\mathrm{HE}>0.5$ indicates persistent long memory in the time series, $\mathrm{HE}<0.5$ implies an anti-correlated time series, and $\mathrm{HE}=0.5$ reflects a random white-noise time series (Gentili et al., 2015). Therefore, Time series can be divided into three categories (Figure 1). Existed fMRI study (Maxim et al., 2005) showed that HE of voxels in gray matter is about 0.8 , and voxels with $\mathrm{HE}<0.5$ concentrate in cerebrospinal fluid. With the characteristics above, $\mathrm{HE}$ is believed to have the capacity to measure brain activity complexity. A higher HE corresponds to a lower fractal dimension, and higher HE means lower complexity accordingly.

\section{Age Effect of HE Based on AAL Brain Atlas}

Here we applied Automated Anatomical Labeling (AAL) brain atlas (Tzourio-Mazoyer et al., 2002) to calculate regional HE.
This atlas consists of a parcellation of 90 brain regions which is normalized to Montreal Neurological Institute coordinates (MNI coord.) space, and distributed by WFU Pickatlas (Maldjian et al., 2003) software.

The voxel-wise HE were averaged in each of 90 brain regions so that we got the region-wise HE of all the regions for all the subjects. In each brain region, Pearson correlation coefficient was used to measure the correlation between the acquired $\mathrm{HE}$ and age. Then, these results were adjusted for multiple comparisons using a False Discovery Rate (FDR) threshold of $q<0.05$.

\section{Gender Comparison Based on AAL Brain Atlas}

The brain cortex was divided into 7 lobar regions including the frontal lobe, insula, limbic lobe, occipital lobe, parietal lobe, sub cortical gray nuclei, and temporal lobe with 90 AAL regions. Values of HE for male and female participants were compared in each lobar region using 2 -tailed $t$-test. Then, further assessments were performed on each AAL region within the lobes where there were significant gender differences. These results were adjusted for multiple comparisons using a FDR threshold of $q<$ 0.05 .

\section{Age Effect of HE Based on Voxel-Wise Analyses}

Statistics analyses were performed using the REST. Linear agerelated effect was estimated by calculating the Pearson correlation coefficient between age and $\mathrm{HE}$ within each brain voxels. Significant activations were detected with the cluster size of at
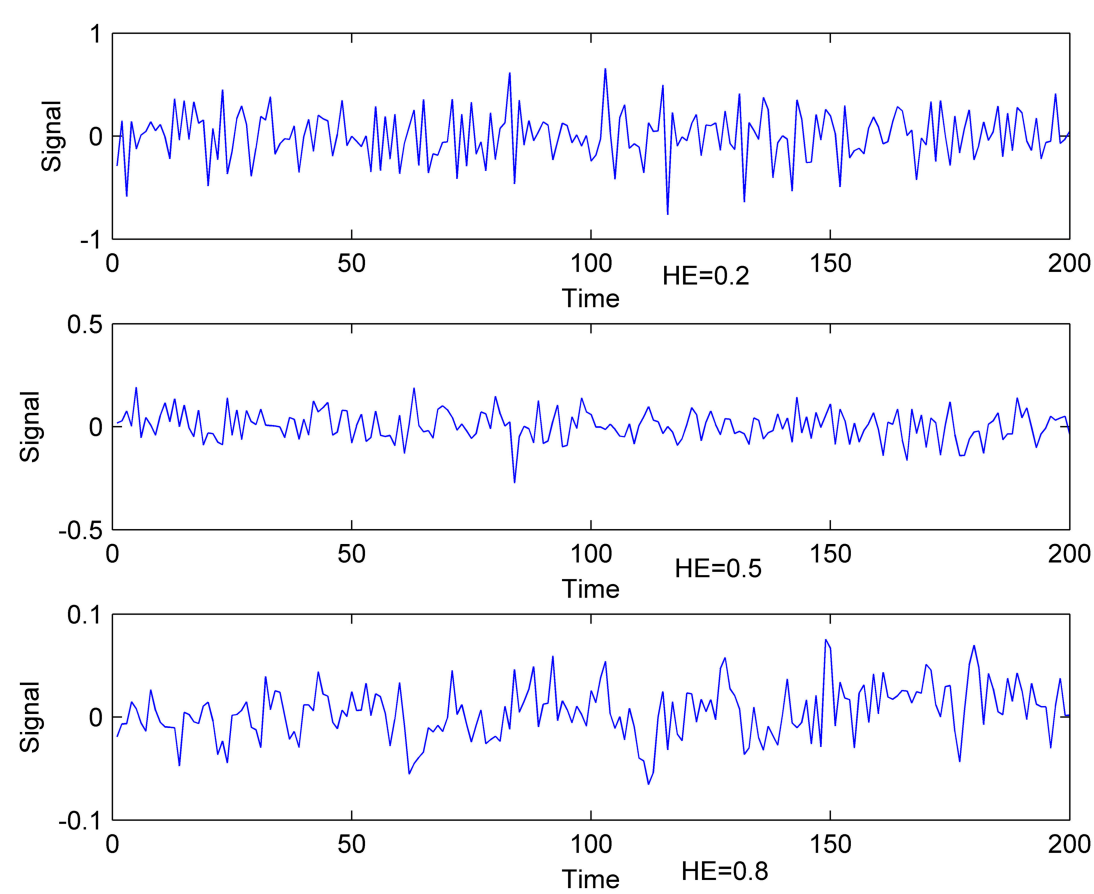

FIGURE 1 | Exemplificative illustrations of time-series with different HE. Lower: time- series with HE of 0.8; Middle: time-series with HE of 0.5; Upper: time-series with HE of 0.2 . 
lowest 20 voxels, and these results were adjusted for multiple comparisons using a FDR threshold of $q<0.05$.

\section{Gender Comparison Based on Voxel-Wise Analyses}

HEs of each of the voxels for males and females were compared using 2-tailed $t$-test. Significant gender differences were detected with the cluster size of at lowest 20 voxels, and these results were adjusted for multiple comparisons using a FDR threshold of $q<0.05$.

\section{Interaction between the Age and Gender}

The regions with significant age effect of $\mathrm{HE}$ were defined as regions of interest (ROIs), and then interaction between the age and gender was assessed within the ROIs.

\section{RESULTS}

For all age effect analyses, gender was entered as covariate of interest. The mean $\mathrm{HE}$ of whole brain gray matter showed significant positive correlation $(r=0.35, p<0.01)$ with age which indicated that complexity of BOLD activity decreased with normal aging.

\section{Age Effect of HE Based on AAL Brain Atlas}

Figure 2 depicts that 33 of the 90 brain regions show less complexity with increasing age. Positive age effect was most significant in parietal lobe, including left angular gyrus $(r=0.21$, $p=0.03$, corrected) and left superior parietal gyrus $(r=0.17$, $p=0.04$, corrected). Significant negative age effect was observed in right insula ( $r=-0.21, p=0.03$, corrected), and in limbic lobe including left parahippocampal gyrus $(r=-0.25, p=0.03$, corrected) and right parahippocampal gyrus $(r=-0.21, p=0.03$, corrected). Also, significant negative age effect was observed in temporal lobe, including left superior temporal gyrus $(r=-0.20$, $p=0.03$, corrected), left superior temporal pole $(r=-0.20$, $p=0.03$, corrected $)$, and right superior temporal pole $(r=-0.23$, $p=0.03$, corrected).

Scatter plots illustrating significant correlations between age and the HE of the AAL regions are shown in Figure 3.

\section{Gender Effect of HE Based on AAL Brain Atlas}

The overall mean HEs of males and females were significantly different $(p=0.04)$. Then, the differences in HE between genders were further assessed in the 7 lobar brain regions, and we found significant differences between genders in parietal lobe $(p=0.04$, corrected) where males had smaller HE (see Figure 4).

Furthermore, an examination of the AAL sub regions in parietal lobe was taken. The results showed smaller male HE in the regions listed in Table 2.

\section{Age Effect of HE Based on Voxel-Wise Analysis}

There is significant positive age effect in frontal and parietal lobe, including left middle frontal gyrus, left triangular part of the inferior frontal gyrus, bilateral superior parietal gyri, bilateral inferior parietal but supramarginal and angular gyri and left angular gyrus. Meanwhile, negative age effects in insula, bilateral parahippocampal gyri,

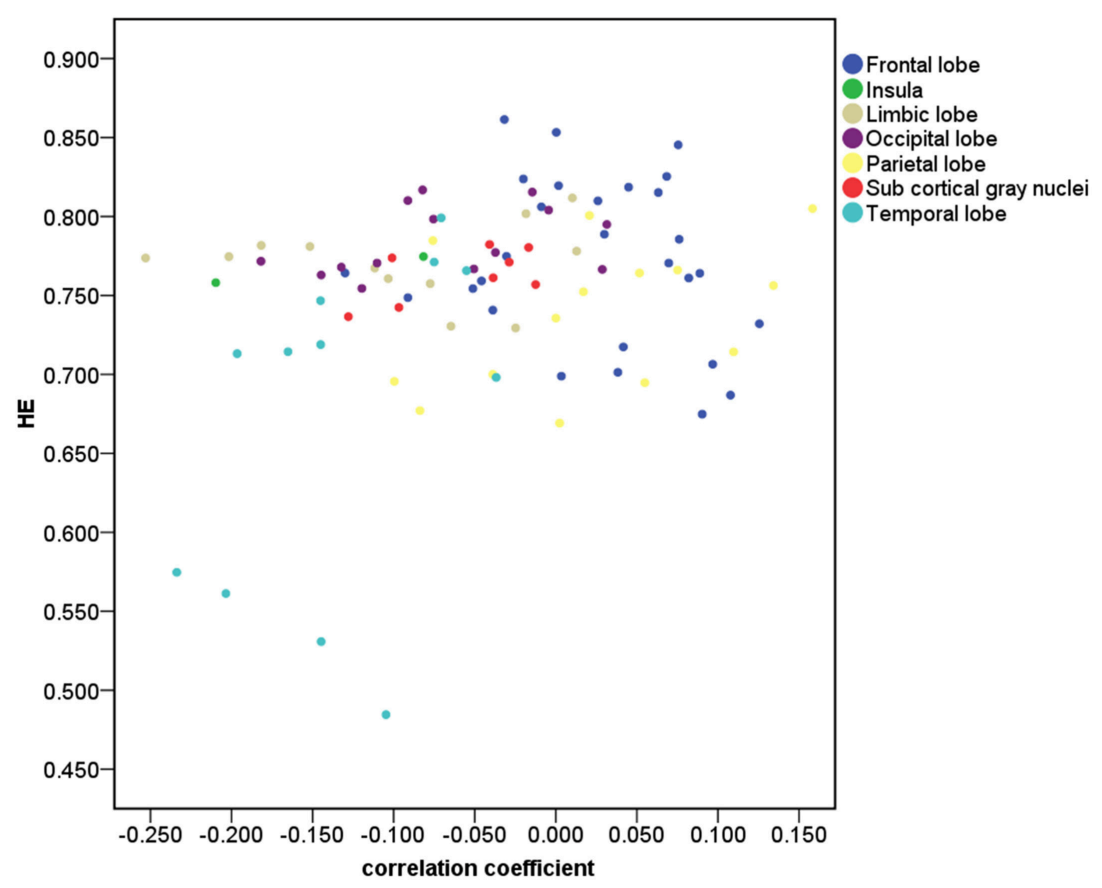

FIGURE 2 | Correlation for the 90 brain regions between HE and age. The brain gray matter was subdivided into 7 lobar regions based on the 90 AAL regions. 

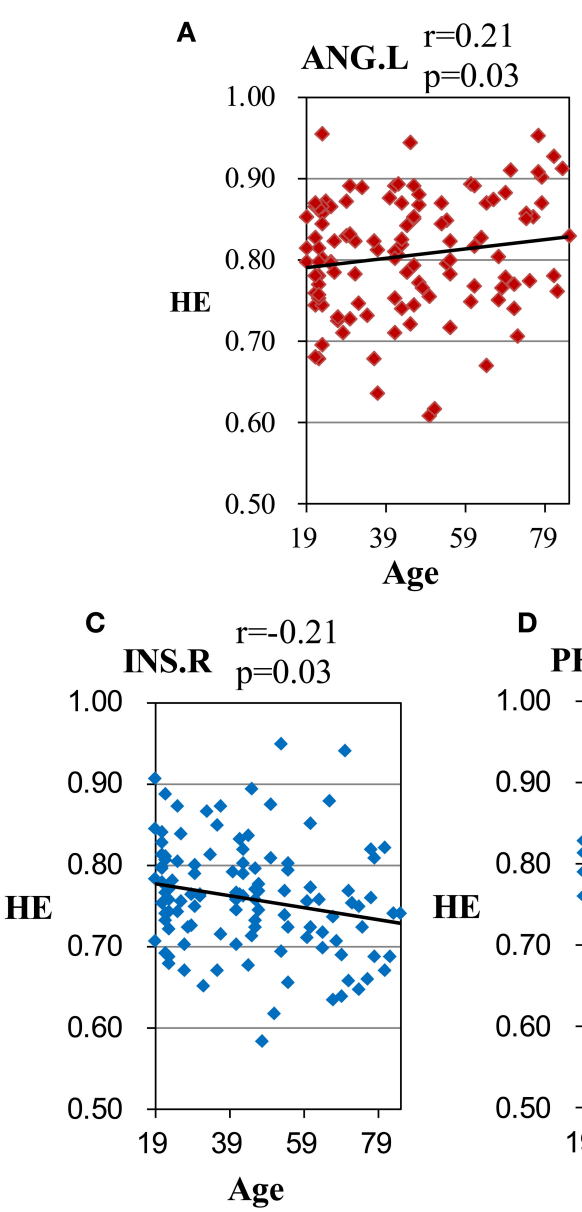

$\mathbf{F}$

$r=-0.20$

STG.L $\mathrm{p}=0.03$

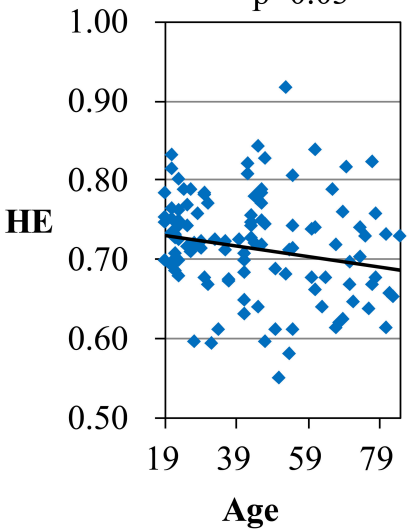

HE

D
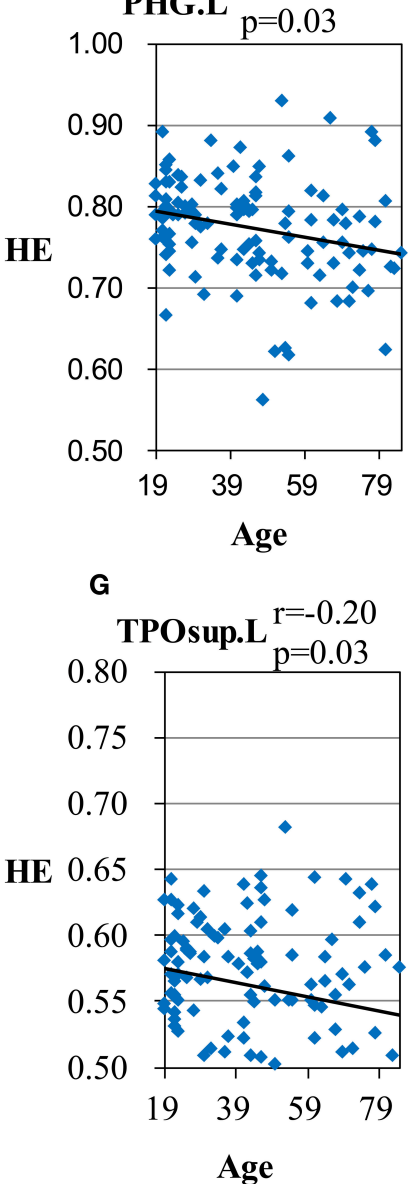

$r=0.17$

SPG.L $\mathrm{p}=0.04$

HE

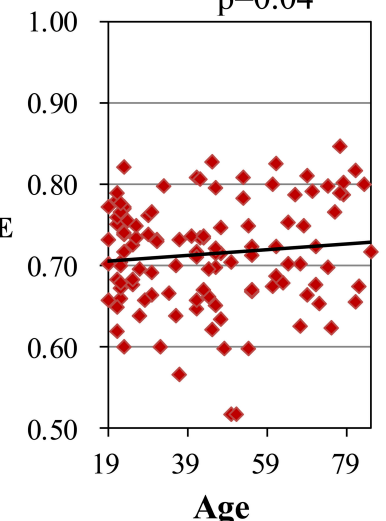

E

$\mathrm{r}=-0.21$

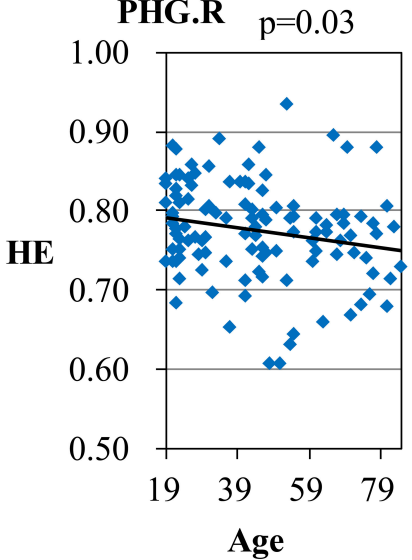

H

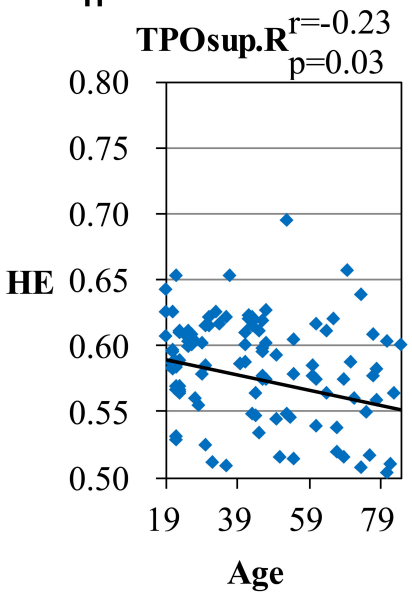

FIGURE 3 | Scatter plots for the significant correlations between age and the HE of the AAL regions. (A) ANG.L, left angular gyrus; (B) SPG.L, left superior parietal gyrus; (C) INS.R, right insula; (D) PHG.L, left parahippocampal gyrus; (E) PHG.R, right parahippocampal gyrus; (F) STG.L, left superior temporal gyrus; (G) TPOsup.L, left superior temporal pole; (H) TPOsup.R, right superior temporal pole. X-axis, age; Y-axis, the mean HE of the AAL regions. The $p$-values were corrected using the FDR method.

bilateral fusiform gyri, and temporal lobe were found. All results are illustrated in Figure 5 and Table 3, where the positive $r$ values indicate a decrease of complexity with age.

\section{Gender Effect of HE Based on Voxel-Wise Analysis}

Differences in HE between genders were assessed in the whole brain gray matter voxels and significant differences were found 


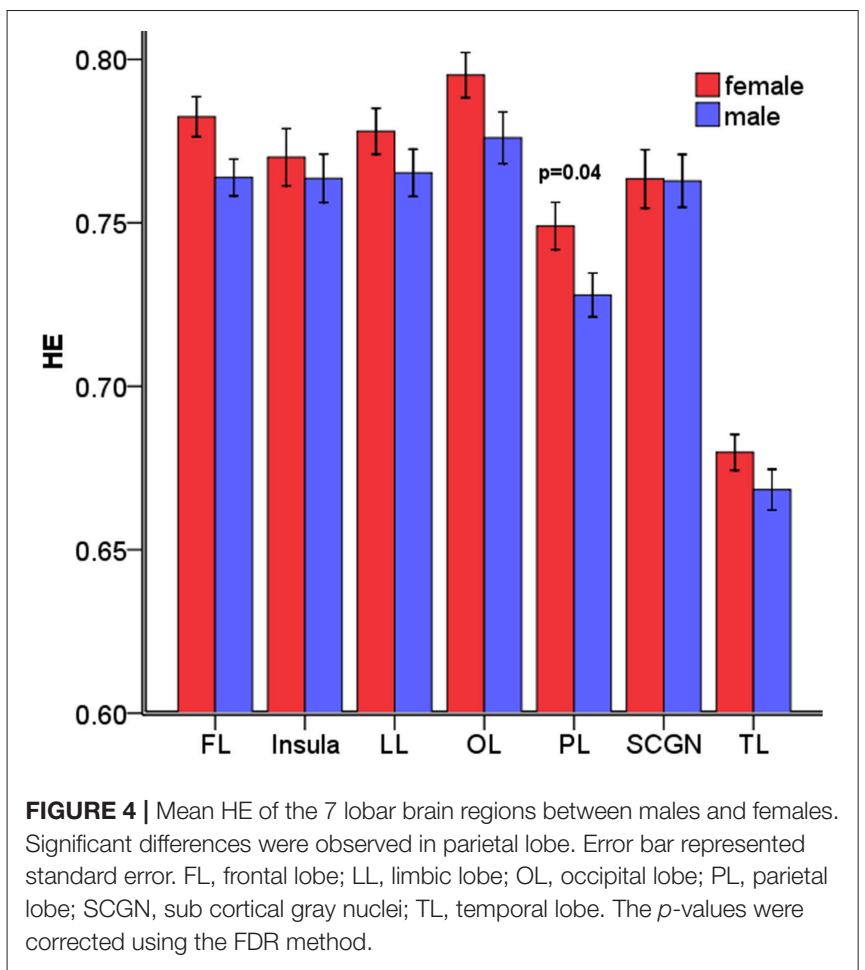

in voxels of frontal lobe, limbic lobe, occipital lobe and parietal lobe ( $p=0.01$, corrected) (see Figure 6 and Table 4 ).

\section{Interaction between the Age and Gender}

Only in voxel-wise analysis, a statistically significant interaction between the age and gender was found in right parahippocampal gyrus $(F=1.89, p=0.04)$, but there was no interaction in the other regions.

\section{DISCUSSION}

Rs-fMRI is based on low frequency fluctuations in the BOLD signal, and these fluctuations arise primarily from endogenous oscillations of brain metabolism and neurophysiological activity (Fox and Raichle, 2007; Yan et al., 2009). The complexity of resting-state BOLD signals could provide some evidence of dynamics of intrinsic brain activity (Yang et al., 2013). In this study, we quantified the complexity of rs-fMRI based on HE analysis in a sample of healthy male and female subjects between the ages of 19-85 years old, and found that there existed a significant $(p<0.01)$ positive correlation $(r=0.35)$ between the mean HE of whole brain gray matter and the age of all subjects which means HE increases with age, that is to say, complexity of BOLD activity is reduced with age. Normal aging is accompanied by a loss of complexity in various physiological processes (Lipsitz, 2004; Yang et al., 2013), and aging was found to be associated with significant decrease of complexity in bilateral hippocampus (Wink et al., 2006). Furthermore, aging may facilitate the erosion of both local and long-range connections in the brain, so it could
TABLE 2 | Differences in HE between genders in the AAL sub regions.

\begin{tabular}{llll}
\hline AAL regions & Side & T & p-values \\
\hline Postcentral gyrus & $\mathrm{L}$ & 2.59 & 0.04 \\
Postcentral gyrus & $\mathrm{R}$ & 2.57 & 0.03 \\
Superior parietal gyrus & $\mathrm{R}$ & 1.76 & 0.04 \\
Inferior parietal but supramarginal and angular gyri & $\mathrm{R}$ & 2.01 & 0.04 \\
Precuneus & $\mathrm{L}$ & 1.89 & 0.04 \\
\hline
\end{tabular}

$L$, left; $R$, right; $T$, Student's t-test. The $p$-values were corrected using the FDR method.

decrease the complexity of spontaneous brain activity (Smith et al., 2014).

In this study, we combined region-wise analysis and voxelwise analysis to explore complexity of resting-state BOLD signals. The region-wise analysis where the mean of all voxels in one region is given cannot reveal brain regional heterogeneity, and can lead to misinterpretation when opposing effects come out from a single structure. However, the voxel-wise analysis often abide a great many voxels to be computed which provides a lot of false positive results (Lebenberg et al., 2011). Therefore, regionwise analysis and voxel-wise analysis are good complementary tools in our study.

With the region-wise analysis, we found age-related loss of complexity in parietal lobe, specifically the left angular gyrus, and left superior parietal gyrus. With the voxel-wise analysis, on the one hand, we also found this decreased complexity in parietal lobe. On the other hand, we further found age-related loss of complexity in frontal lobe with the voxel-wise analysis, specifically left middle frontal gyrus, and triangular part of the inferior frontal gyrus. With respect to age-related characteristics of complexity of resting-state BOLD signals, some studies used different metrics to investigate characteristics of complexity in brain regions. Liu et al. found decreased complexity in the right middle temporal gyrus, bilateral angular gyri, left middle, and posterior cingulate, left supramarginal gyrus, and left calcarine cortex in aged subjects compared to young subjects (Liu et al., 2013). Compared to Liu et al.'s study, we also found decreased complexity in left angular gyrus. However, we found inconsistent results in right mid temporal gyrus, left mid cingulate, and left calcarine cortex of this study. This may due to the difference of sample size ( $n=116$ in our study vs. $n=16$ in their study) and age range (19-85 years old vs. two groups, young: age $23 \pm 2$ and elderly: age $66 \pm 3$ ). And what's more, we performed correlation analyses between the HE and age to investigate the age effect on complexity, however, Liu et al. used approximate entropy as a measure of complexity in two groups of healthy subjects consisting of old and young volunteers. Our results about agerelated decrease of complexity is most pronounced in parietal and frontal lobe, which is consistent with the regions found by Sokunbi et al.'s study (Sokunbi et al., 2015) where the age range of subjects is similar with ours, and they measured rs-fMRI signal complexity utilizing fuzzy approximate entropy.

Regarding the mechanism for age-related loss of complexity in these brain regions, we know that inferior frontal gyrus is integral for language function and the mirror system (Lai et al., 2010) and 


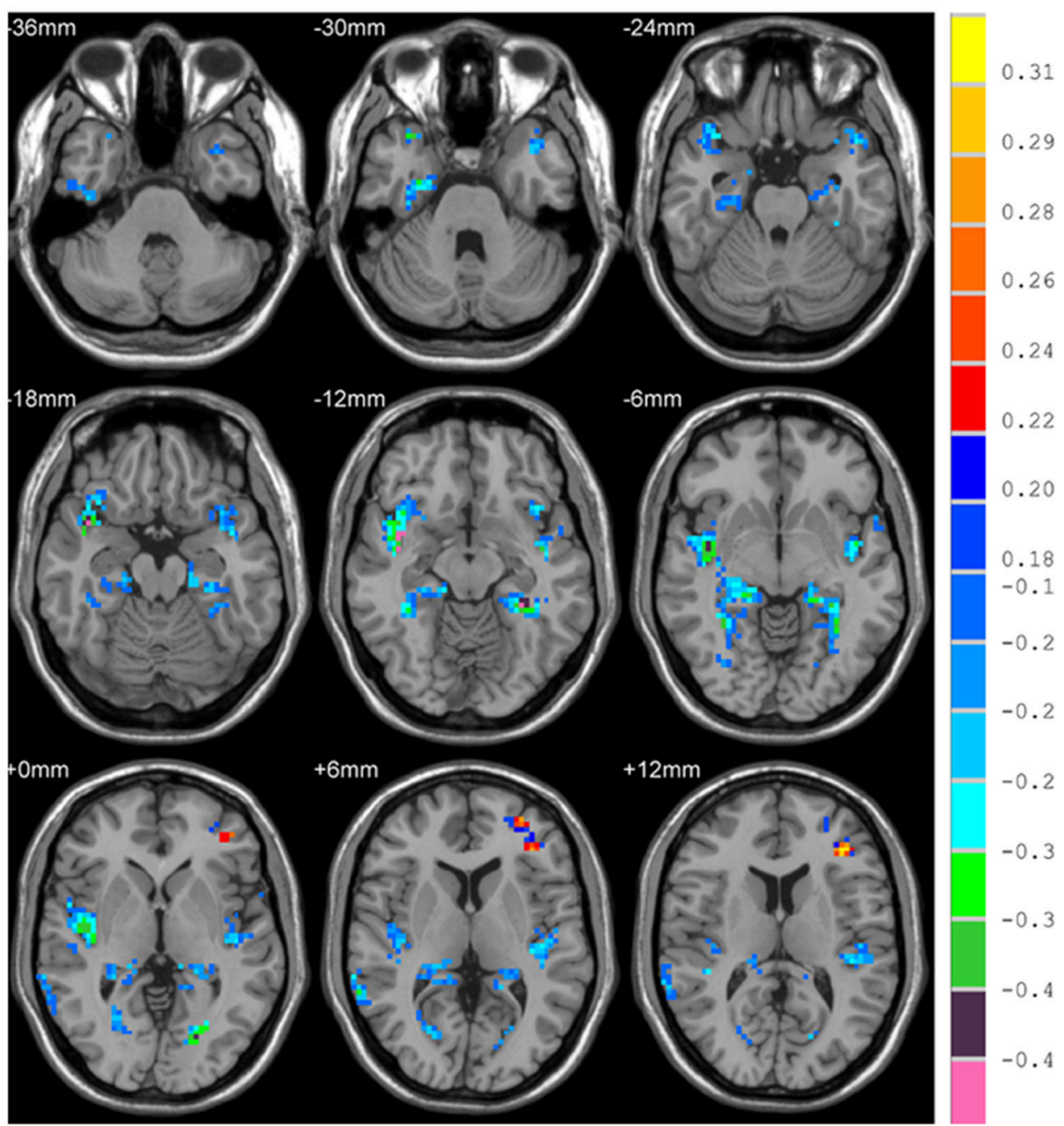

FIGURE 5 | Correlation between HE and age, with positive values showing a decrease of complexity with age.

TABLE 3 | Correlation between HE and age.

\begin{tabular}{|c|c|c|c|c|c|c|}
\hline \multirow[t]{2}{*}{ AAL regions } & \multirow[t]{2}{*}{ Side } & \multirow[t]{2}{*}{ Cluster size } & \multicolumn{3}{|c|}{ Peak MNI coord. (mm) } & \multirow[t]{2}{*}{$r$} \\
\hline & & & $\mathbf{x}$ & $\mathbf{Y}$ & $\mathbf{Z}$ & \\
\hline Insula, ParaHippocampal, Fusiform, Temporal_Pole_Sup, Calcarine & $\mathrm{R}$ & 765 & 42 & 9 & -18 & -0.47 \\
\hline Temporal_Sup, Temporal_Pole_Sup/Mid, Insula & $\mathrm{L}$ & 267 & -42 & -9 & -9 & -0.43 \\
\hline ParaHippocampal, Fusiform, Calcarine & $L$ & 304 & -30 & -39 & -12 & -0.43 \\
\hline Temporal_Mid/Sup & $\mathrm{R}$ & 77 & 66 & -45 & 9 & -0.35 \\
\hline Frontal_Inf_Tri, Frontal_Mid/Sup & $\mathrm{L}$ & 103 & -36 & 36 & 12 & 0.33 \\
\hline Parietal_Inf/Sup, Angular & $L$ & 128 & -36 & -54 & 63 & 0.31 \\
\hline Parietal_Inf/Sup & $\mathrm{R}$ & 48 & 36 & -60 & 60 & 0.28 \\
\hline
\end{tabular}

Temporal_Pole_Sup, superior temporal pole; Temporal_Sup, superior temporal gyrus; Temporal_Pole_Mid, middle temporal pole; Temporal_Mid, middle temporal gyrus; Frontal_Inf_Tri,

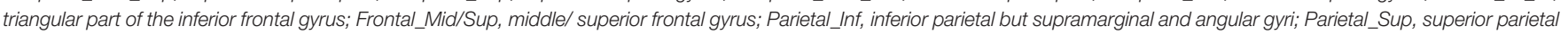
gyrus; L, left; $R$, right; $r$, Pearson correlation coefficient. 


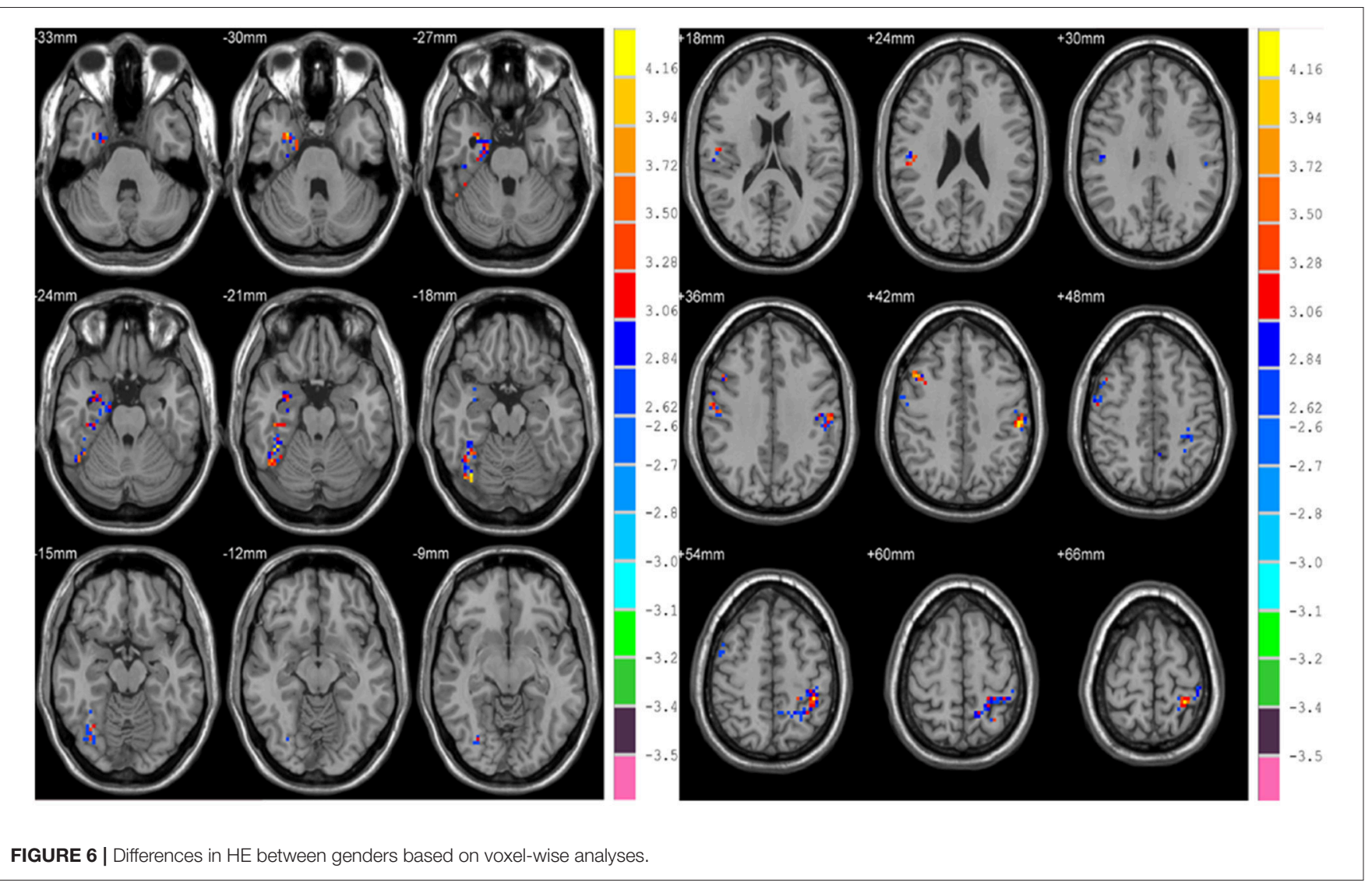

TABLE 4 | Differences in HE between genders.

\begin{tabular}{|c|c|c|c|c|c|c|}
\hline \multirow[t]{2}{*}{ AAL regions } & \multirow[t]{2}{*}{ Side } & \multirow[t]{2}{*}{ Cluster size } & \multicolumn{3}{|c|}{ Peak MNI coord. (mm) } & \multirow[t]{2}{*}{$T$} \\
\hline & & & $\mathbf{x}$ & $\mathbf{Y}$ & $\mathbf{Z}$ & \\
\hline ParaHippocampal, Hippocampus & $\mathrm{R}$ & 79 & 30 & 0 & -30 & 4.16 \\
\hline Fusiform, Temporal_Inf & $\mathrm{R}$ & 77 & 36 & -69 & -18 & 4.34 \\
\hline Frontal_Mid, Postcentral, Precentral & $\mathrm{R}$ & 108 & 45 & 15 & 42 & 4.10 \\
\hline Parietal_Inf, SupraMarginal, Postcentral & $\mathrm{L}$ & 55 & -54 & -24 & 42 & 4.29 \\
\hline Parietal_Sup, Precuneus & $\mathrm{L}$ & 169 & -39 & -39 & 54 & 4.21 \\
\hline
\end{tabular}

Temporal_Inf, inferior temporal gyrus; Frontal_Mid, middle frontal gyrus; Parietal_Inf, inferior parietal but supramarginal and angular gyri; Parietal_Sup, superior parietal gyrus; L, left; R, right; T, Student's t-test.

important for cognitive control (Schlesinger et al., 2017). It has been suggested that cognitive control is modulated by age (Treitz et al., 2007). Our results about age-related loss of complexity are in agreement with the Lipsitz model which demonstrated healthier system exhibits more complexly in their physiological output and complexity of system decreases with age (Lipsitz, 2004).

On the contrary, we found that age-related increase of complexity is most pronounced in insula, limbic lobe (bilateral parahippocampal gyri), and temporal lobe (left superior temporal gyrus, bilateral superior temporal poles) using two level analyses. The insula plays a vital role in interactions between motor, affective, and cognitive functions (Mathys et al., 2014). We speculate age-related increase of complexity in insula is due to that insula is critical for emotional feeling (Gasquoine, 2014), and with aging, the adult's ability to regulate emotion remains stable and improves in some aspects (Nashiro et al., 2012). Superior temporal gyrus and temporal pole are necessary for understanding perspective taking, movements, and convergence of social knowledge (Lai et al., 2010). In addition, with the voxel-wise analysis, age-related increase of complexity was found in fusiform gyrus and right middle temporal gyrus. Generally, fusiform gyrus is related to cognitive functions (SchenkerAhmed and Annese, 2013). Complexity increased with age in these regions which suggested that potential compensatory mechanisms may play a role (Sugiura, 2016). 
Brain aging may differ between genders. In our study, differences in HE between genders were found to be significant in the parietal lobe (bilateral postcentral gyri and left inferior parietal but supramarginal and angular gyri) on both regionwise and voxel-wise analyses, with females exhibiting higher HE values. In addition, voxel-wise based analysis showed that there were significant differences between genders in the right parahippocampal gyrus and fusiform gyrus. The inferior parietal lobule is a part of attention network, and parahippocampal gyrus is correlated with short-term memory. We conjecture that gender differences occur in the behavioral and cognitive domains because women generally excel in language (Hyde and Linn, 1988), emotional memories (Canli et al., 2002), and facial emotion recognition (Rahman et al., 2004).

In addition, we tested the interaction between the age and gender in terms of $\mathrm{HE}$ and found an interaction in right parahippocampal gyrus $(p=0.04)$, suggesting that the age effect on complexity was different between genders in this region.

There are several limitations in our study. We only focused on functional changes without including any structural analysis which could provide a broader view of age-related brain changes by considering structural differences and their associations with functional results. Moreover, cortical atrophy makes spatial normalization difficult for older subjects, and can potentially decrease the signal to noise ratio of the data. Therefore, further investigations are needed to confirm current findings.

\section{ETHICS STATEMENT}

Data used in preparation of this article were obtained from the International Neuroimaging Data-sharing Initiative (INDI) online database (http://fcon_1000.projects.nitrc.org/indi/

\section{REFERENCES}

Abrahamson, K., Clark, D., Perkins, A., and Arling, G. (2012). Does cognitive impairment influence quality of life among nursing home residents? Gerontologist 52, 632-640. doi: 10.1093/geront/gnr137

Bandettini, P. (2007). Functional MRI today. Int. J. Psychophysiol. 63, 138-145. doi: 10.1016/j.ijpsycho.2006.03.016

Boghi, A., Rasetti, R., Avidano, F., Manzone, C., Orsi, L., D’Agata, F., et al. (2006). The effect of gender on planning: an fMRI study using the Tower of London task. Neuroimage 33, 999-1010. doi: 10.1016/j.neuroimage.2006.07.022

Canli, T., Desmond, J. E., Zhao, Z., and Gabrieli, J. D. E. (2002). Sex differences in the neural basis of emotional memories. Proc. Natl. Acad. Sci. U.S.A. 99:10789. doi: 10.1073/pnas.162356599

Chen, J. E., and Glover, G. H. (2015). BOLD fractional contribution to resting-state functional connectivity above $0.1 \mathrm{~Hz}$. Neuroimage 107, 207-218. doi: 10.1016/j.neuroimage.2014.12.012

Ciuciu, P., Varoquaux, G., Abry, P., Sadaghiani, S., and Kleinschmidt, A. (2012). Scale-free and multifractal time dynamics of fMRI signals during rest and task. Front. Physiol. 3:186. doi: 10.3389/fphys.2012.00186

Dosenbach, N. U. F., Nardos, B., Cohen, A. L., Fair, D. A., Power, J. D., Church, J. A., et al. (2010). Prediction of individual brain maturity using fMRI. Science 329, 1358-1361. doi: 10.1126/science.1194144

Expert, P., Lambiotte, R., Chialvo, D. R., Christensen, K., Jensen, H. J., Sharp, D. J., et al. (2011). Self-similar correlation function in brain resting-state functional magnetic resonance imaging. J. R. Soc. Interface 8, 472-479. doi: $10.1098 /$ rsif.2010.0416 pro/nki.html). As such, the investigators within the INDI contributed to the design and implementation of INDI and/or provided data but did not participate in analysis or writing of this report.

\section{AUTHOR CONTRIBUTIONS}

JD made substantial contributions to the conception and design of the work, analysis and interpretation of data for the work, and the draft of the manuscript; $\mathrm{BJ}$ and XM made a contribution to the revision of the manuscript; HLiu and XM made a contribution to the conception and design of the work. As the corresponding author, HLi made great contributions to interpretation of data, and determined the final version to be published. All authors have read and approved the final manuscript.

\section{FUNDING}

This work was supported by National Natural Science Foundation of China [grant number 81220108007]; Beijing Natural Science Foundation [grant number 4122018]; and Beijing Natural Science Foundation [grant number 7174282].

\section{ACKNOWLEDGMENTS}

The authors are willing to acknowledge the Nathan Kline Institute (NKI) fMRI community for creating the resting-state database and making it publicly available at the International Neuroimaging Data-sharing Initiative online database ${ }^{4}$.

${ }^{4}$ http://fcon_1000.projects.nitrc.org/indi/pro/nki.html
Fox, M. D., and Raichle, M. E. (2007). Spontaneous fluctuations in brain activity observed with functional magnetic resonance imaging. Nat. Rev. Neurosci. 8, 700-711. doi: 10.1038/nrn2201

Friston, K. J., Holmes, A., Poline, J. B., Price, C. J., and Frith, C. D. (1996). Detecting activations in PET and fMRI: levels of inference and power. Neuroimage 4, 223-235. doi: 10.1006/nimg.1996.0074

Gasquoine, P. G. (2014). Contributions of the insula to cognition and emotion. Neuropsychol. Rev. 24, 77-87. doi: 10.1007/s11065-014-9246-9

Gentili, C., Vanello, N., Cristea, I., David, D., Ricciardi, E., and Pietrini, P. (2015). Proneness to social anxiety modulates neural complexity in the absence of exposure: a resting state fMRI study using Hurst exponent. Psychiatry Res. 232, 135-144. doi: 10.1016/j.pscychresns.2015.03.005

Grady, C. L. (2008). Cognitive neuroscience of aging. Ann. N. Y. Acad. Sci. 1124, 127-144. doi: 10.1196/annals. 1440.009

He, B. J. (2014). Scale-free brain activity: past, present, and future. Trends Cogn. Sci. 18, 480-487. doi: 10.1016/j.tics.2014.04.003

Hedden, T., and Gabrieli, J. D. E. (2004). Insights into the ageing mind: a view from cognitive neuroscience. Nat. Rev. Neurosci. 5, 87-96. doi: 10.1038/nrn1323

Hofer, A., Siedentopf, C. M., Ischebeck, A., Rettenbacher, M. A., Verius, M., Felber, S., et al. (2006). Gender differences in regional cerebral activity during the perception of emotion: a functional MRI study. Neuroimage 32, 854-862. doi: 10.1016/j.neuroimage.2006.03.053

Hurst, H. E. (1951). Long term storage capacity of reservoirs. Trans. Am. Soc. Civil Eng. 116, 776-808.

Hyde, J. S., and Linn, M. C. (1988). Gender differences in verbal ability: a meta-analysis. Psychol. Bull. 104, 53-69. doi: 10.1037/0033-2909.104.1.53 
Keller, K., and Menon, V. (2009). Gender differences in the functional and structural neuroanatomy of mathematical cognition. Neuroimage 47, 342-352. doi: 10.1016/j.neuroimage.2009.04.042

Kruger, G., and Glover, G. H. (2001). Physiological noise in oxygenationsensitive magnetic resonance imaging. Magn. Reson. Med. 46, 631-637. doi: $10.1002 / \mathrm{mrm} .1240$

Lai, M. C., Lombardo, M. V., Chakrabarti, B., Sadek, S. A., Pasco, G., Wheelwright, S. J., et al. (2010). A shift to randomness of brain oscillations in people with autism. Biol. Psychiatry 68, 1092-1099. doi: 10.1016/j.biopsych.2010.06.027

Lebenberg, J., Herard, A. S., Dubois, A., Dhenain, M., Hantraye, P., and Delzescaux, T. (2011). A combination of atlas-based and voxel-wise approaches to analyze metabolic changes in autoradiographic data from Alzheimer's mice. Neuroimage 57, 1447-1457. doi: 10.1016/j.neuroimage.2011.04.059

Lipsitz, L. A. (2004). Physiological complexity, aging, and the path to frailty. Sci. Aging Knowledge Environ. 2004:pe16. doi: 10.1126/sageke.2004.16.pe16

Liu, C. Y., Krishnan, A. P., Yan, L., Smith, R. X., Kilroy, E., Alger, J. R., et al. (2013). Complexity and synchronicity of resting state blood oxygenation leveldependent (BOLD) functional MRI in normal aging and cognitive decline. J. Magn. Reson. Imaging 38, 36-45. doi: 10.1002/jmri.23961

Lopez-Larson, M. P., Anderson, J. S., Ferguson, M. A., and Yurgelun-Todd, D. (2011). Local brain connectivity and associations with gender and age. Dev. Cogn. Neurosci. 1, 187-197. doi: 10.1016/j.dcn.2010.10.001

Maldjian, J. A., Laurienti, P. J., Kraft, R. A., and Burdette, J. H. (2003). An automated method for neuroanatomic and cytoarchitectonic atlasbased interrogation of fMRI data sets. Neuroimage 19, 1233-1239. doi: 10.1016/S1053-8119(03)00169-1

Mathys, C., Hoffstaedter, F., Caspers, J., Caspers, S., Sudmeyer, M., Grefkes, C., et al. (2014). An age-related shift of resting-state functional connectivity of the subthalamic nucleus: a potential mechanism for compensating motor performance decline in older adults. Front. Aging Neurosci. 6:178. doi: 10.3389/fnagi.2014.00178

Maxim, V., Sendur, L., Fadili, J., Suckling, J., Gould, R., Howard, R., et al. (2005). Fractional Gaussian noise, functional MRI and Alzheimer's disease. Neuroimage 25, 141-158. doi: 10.1016/j.neuroimage.2004.10.044

Mcrae, K., Ochsner, K. N., Mauss, I. B., Gabrieli, J. J. D., and Gross, J. J. (2008). Gender differences in emotion regulation: an fMRI study of cognitive reappraisal. Group Processes Intergroup Relations 11, 143-162. doi: $10.1177 / 1368430207088035$

Nashiro, K., Sakaki, M., and Mather, M. (2012). Age differences in brain activity during emotion processing: reflections of age-related decline or increased emotion regulation? Gerontology 58:156. doi: 10.1159/0003 28465

Ni, H., Huang, X., Ning, X., Huo, C., Liu, T., and Ben, D. (2014). Multifractal analysis of resting state fMRI series in default mode network: age and gender effects. Chin. Sci. Bull. 59, 3107-3113. doi: 10.1007/s11434-0140355-x

Nooner, K. B., Colcombe, S. J., Tobe, R. H., Mennes, M., Benedict, M. M., Moreno, A. L., et al. (2012). The NKI-Rockland sample: a model for accelerating the pace of discovery science in psychiatry. Front. Neurosci. 6:152. doi: 10.3389/fnins.2012.00152

Park, C., Lazar, N. A., Ahn, J., and Sornborger, A. (2010). A multiscale analysis of the temporal characteristics of resting-state fMRI data. J. Neurosci. Methods 193, 334-342. doi: 10.1016/j.jneumeth.2010.08.021

Rahman, Q., Wilson, G. D., and Abrahams, S. (2004). Sex, sexual orientation, and identification of positive and negative facial affect. Brain Cogn. 54, 179-185. doi: 10.1016/j.bandc.2004.01.002

Schenker-Ahmed, N. M., and Annese, J. (2013). Cortical mapping by magnetic resonance imaging (MRI) and quantitative cytological analysis in the human brain: a feasibility study in the fusiform gyrus. J. Neurosci. Methods 218, 9-16. doi: 10.1016/j.jneumeth.2013.04.018

Schlesinger, K. J., Turner, B. O., Lopez, B. A., Miller, M. B., and Carlson, J. M. (2017). Age-dependent changes in task-based modular organization of the human brain. Neuroimage 146, 741-762. doi: 10.1016/j.neuroimage.2016.09.001

Schulterüther, M., Markowitsch, H. J., Shah, N. J., Fink, G. R., and Piefke, M. (2008). Gender differences in brain networks supporting empathy. Neuroimage 42:393. doi: 10.1016/j.neuroimage.2008.04.180

Smith, R. X., Yan, L., and Wang, D. J. (2014). Multiple time scale complexity analysis of resting state FMRI. Brain Imaging Behav. 8, 284-291. doi: 10.1007/s11682-013-9276-6

Sokunbi, M. O., Cameron, G. G., Ahearn, T. S., Murray, A. D., and Staff, R. T. (2015). Fuzzy approximate entropy analysis of resting state fMRI signal complexity across the adult life span. Med. Eng. Phys. 37, 1082-1090. doi: 10.1016/j.medengphy.2015.09.001

Song, X. W., Dong, Z. Y., Long, X. Y., Li, S. F., Zuo, X. N., Zhu, C. Z., et al. (2011). REST: a toolkit for resting-state functional magnetic resonance imaging data processing. PLoS ONE 6:e25031. doi: 10.1371/journal.pone.0025031

St John, P. D., and Montgomery, P. R. (2010). Cognitive impairment and life satisfaction in older adults. Int. J. Geriatr. Psychiatry 25, 814-821. doi: $10.1002 /$ gps.2422

Sugiura, M. (2016). Functional neuroimaging of normal aging: declining brain, adapting brain. Ageing Res. Rev. 30, 61-72. doi: 10.1016/j.arr.2016.02.006

Treitz, F. H., Heyder, K., and Daum, I. (2007). Differential course of executive control changes during normal aging. Neuropsychol. Dev. Cogn. B Aging Neuropsychol. Cogn. 14, 370-393. doi: 10.1080/13825580600678442

Tzourio-Mazoyer, N., Landeau, B., Papathanassiou, D., Crivello, F., Etard, O., Delcroix, N., et al. (2002). Automated anatomical labeling of activations in SPM using a macroscopic anatomical parcellation of the MNI MRI single-subject brain. Neuroimage 15, 273-289. doi: 10.1006/nimg.2001.0978

Uddin, L. Q., Supekar, K., and Menon, V. (2010). Typical and atypical development of functional human brain networks: insights from resting-state fMRI. Front. Syst. Neurosci. 4:21. doi: 10.3389/fnsys.2010.00021

Wink, A. M., Bernard, F., Salvador, R., Bullmore, E., and Suckling, J. (2006). Age and cholinergic effects on hemodynamics and functional coherence of human hippocampus. Neurobiol. Aging 27, 1395-1404. doi: 10.1016/j.neurobiolaging.2005.08.011

Yan, C. G., Craddock, R. C., Zuo, X. N., Zang, Y. F., and Milham, M. P. (2013). Standardizing the intrinsic brain: towards robust measurement of interindividual variation in 1000 functional connectomes. Neuroimage 80, 246-262. doi: 10.1016/j.neuroimage.2013.04.081

Yan, C.-G., and Zang, Y.-F. (2010). DPARSF: a MATLAB toolbox for "Pipeline" data analysis of resting-state fMRI. Front. Syst. Neurosci. 4:13. doi: 10.3389/fnsys.2010.00013

Yan, L., Zhuo, Y., Ye, Y., Xie, S. X., An, J., Aguirre, G. K., et al. (2009). Physiological origin of low-frequency drift in blood oxygen level dependent (BOLD) functional magnetic resonance imaging (fMRI). Magn. Reson. Med.61, 819-827. doi: 10.1002/mrm.21902

Yang, A. C., Huang, C. C., Yeh, H. L., Liu, M. E., Hong, C. J., Tu, P. C., et al. (2013). Complexity of spontaneous BOLD activity in default mode network is correlated with cognitive function in normal male elderly: a multiscale entropy analysis. Neurobiol. Aging 34, 428-438. doi: 10.1016/j.neurobiolaging.2012.05.004

Conflict of Interest Statement: The authors declare that the research was conducted in the absence of any commercial or financial relationships that could be construed as a potential conflict of interest.

Copyright (c) 2018 Dong, Jing, Ma, Liu, Mo and Li. This is an open-access article distributed under the terms of the Creative Commons Attribution License (CC $B Y)$. The use, distribution or reproduction in other forums is permitted, provided the original author(s) and the copyright owner are credited and that the original publication in this journal is cited, in accordance with accepted academic practice. No use, distribution or reproduction is permitted which does not comply with these terms. 